\title{
Comunicação Cinésica e Proxémica de Instrutores de Localizada Experientes e Estagiários: Deteção de T-patterns
}

\author{
Kinesics and Proxemics Communication of Expert and Novice Group \\ Resistance Fitness Instructors: T-patterns Detection
}

\author{
Susana Mendes Alves ${ }^{1}$, 2, 3*, Marta Castañer ${ }^{4}$, Oleguer Camerino ${ }^{4}$, Susana Carla Alves \\ Franco $^{1,5}$, José Jesus Fernandes Rodrigues ${ }^{1,2,6}$
}

ARTIGO ORIGINAL | ORIGINALARTICLE \begin{abstract}
O presente estudo centra-se na análise da comunicação não-verbal dos instrutores de fitness com recurso à metodologia observacional e objetiva a análise das configurações e padrões temporais ( $T$-patterns) de comportamento cinésico e proxémico de instrutores com diferentes níveis de experiência profissional. Participaram neste estudo 12 instrutores de fitness divididos em dois grupos em função da sua experiência profissional: grupo de instrutores experientes $(n=6)$; grupo de instrutores estagiários $(n=6)$. As sessões de exercício foram filmadas e os comportamentos cinésicos e proxémicos dos instrutores codificados com recurso ao Sistema de Observação da Comunicação Cinésica - Fitness (SOCIN-Fitness) e ao Sistema de Observação da Comunicação Proxémica - Fitness (SOPROX-Fitness). Os resultados obtidos revelaram a existência de padrões temporais de comportamento cinésico e proxémico, próprios de cada grupo de instrutores. Foi possível verificar que os instrutores experientes apresentam um comportamento cinésico e proxémico mais consistente e complexo do que os estagiários, caraterizado pela regulação do comportamento dos praticantes, através do acompanhamento na realização dos exercícios a partir da posição correspondente, e da intercalação de comportamentos de informação e de feedback, com recurso à utilização de uma morfologia de gestos mais diversificadas.
\end{abstract}

Palavras-chave: comunicação não-verbal, metodologia observacional, fitness

\begin{abstract}
The present study focuses on the analyses of the fitness instructors' non-verbal communication using the observational methodology and it aims the analysis of the configuration and temporal pattern (T-patterns) of kinesics and proxemics behaviour, considering different levels of professional experience. 12 fitness instructors participate in this study, divided in two groups accordingly with their professional experience: expert group $(n=6)$; novice group $(n=6)$. The exercise classes were videotaped and fitness instructors' behaviours coded using the Observational System for the Kinesics Communication of the Fitness Instructor (SOCIN-Fitness) and the Observational System for the Proxemics Communication of the Fitness Instructor (SOPROX-Fitness). The results revealed the existence of kinesics and proxemics temporal patterns of communication distinct for each group of instructors. It was possible to verify that expert instructors have a more consistence and complex kinesics and proxemics behaviour than the novices, which was characterized by the regulation of exerciser's behaviour, throughout their participation in exercise from a correspondent position, and the intercalation of behaviours to inform and give feedback, using more diverse morphologies of gestures.
\end{abstract}

Key words: non-verbal communication, observational methodology, fitness

\footnotetext{
Artigo recebido a 06.05.2014; Aceite a 08.02.2015

${ }^{1}$ Escola Superior de Desporto de Rio Maior (ESDRM), Rio Maior

${ }^{2}$ Centro de Investigação em Qualidade de Vida (CIEQV), Santarém

${ }^{3}$ Centro de Investigação em Desporto, Saúde e Desenvolvimento Humano (CIDESD), Vila Real

${ }^{4}$ Institut Nacional d'Educació Física de Catalunya (INEFC) - Universitat de Lleida, Grup de recerca en Metodologia Observacional en l'activitat Física i Esportiva.

${ }^{5}$ Centro de Estudos em Educação, Tecnologia e Saúde (CI\&DETS), Viseu

${ }^{6}$ Laboratório de Biociências da Motricidade Humana (LABIMH), Aguaçu, Brasil

* Autor correspondente: Escola Superior de Desporto de Rio Maior, Instituto Politécnico de Santarém, Av. Mário Soares 110, 2040-413 Rio Maior, Portugal; E-mail: salves@esdrm.ipsantarem.pt
} 


\section{INTRODUÇÃo}

As atividades de grupo de fitness representam uma boa oportunidade para os indivíduos atingirem os níveis de atividade física recomendadas para a saúde e, quando comparadas com as atividades individuais, tendem também a promover mais benefícios psicossociais e mais adesão ao exercício (Carron \& Burke, 2005). Todavia, grande parte destes benefícios depende da qualidade dos instrutores, já que são estes, em última instância, que têm a responsabilidade de organizar e estruturar as atividades. Esta tese é corroborada por alguns estudos que têm demonstrado a existência de uma relação entre a forma como os instrutores comunicam e o clima de aula e a adesão às atividades de grupo de fitness (Bray, Millen, Eidsness, \& Leuzinger, 2005; Martin \& Fox, 2001).

Tendo por base este pressuposto, este estudo centra-se na análise da comunicação cinésica (i.e. movimentos corporais) (Birdwhistell, 1970) e proxémica (i.e. uso do espaço) (Hall, 1966) dos instrutores de fitness de Localizada, considerando a sua experiência profissional. A opção pela análise deste tipo de comunicação em função da experiência profissional tem por base o facto de os indivíduos se irem tornando mais competentes e eficazes na realização das suas atividades à medida que vão ganhando experiência (Berliner, 2001). De acordo com Webster (2008), este tipo de análise comparativa, entre professores com e sem experiência, permite um melhor entendimento sobre o que constitui uma comunicação de sucesso, já que ao se ter acesso aos automatismos caraterísticos dos professores experientes (Berliner, 2001; Sabers, Cushing, \& Berliner, 1991), possibilita-se a aprendizagem dos professores menos experientes (FarringtonDarby \& Wilson, 2006).

Os estudos realizados recentemente no contexto do ensino permitiram identificar a existência de T-patterns de comportamentos de comunicação próprios de professores de educação física com e sem experiência (Castañer,
Camerino, Anguera, \& Jonsson, 2010, 2013). De acordo com estes estudos, os professores com menos experiência, apesar de realizarem uma maior quantidade de gestos, apresentavam uma comunicação gestual de menor qualidade e nem sempre tiravam o melhor partido da ocupação do espaço, influenciando diretamente as aprendizagens dos alunos.

Estes resultados fazem acreditar que o estudo destas variáveis no contexto das atividades de grupo de fitness possa também contribuir para o conhecimento do perfil de comunicação dos instrutores, ajudando-os na otimização do processo de ensino. Tendo por base esta expetativa, o presente estudo objetiva a análise dos T-patterns de comportamento cinésico e proxémico dos instrutores de fitness, na lecionação da atividade de Localizada, em contexto real de atuação profissional.

\section{MÉTODO}

Dada a complexidade e a dinâmica da interação entre os comportamentos comunicativos cinésicos e proxémicos dos instrutores de Localizada, recorreu-se à metodologia observacional (Anguera, 2003), a qual tem como princípios: a implementação em contextos naturais, a elaboração de instrumentos de observação ad hoc, e a continuidade temporal (Anguera, Blanco-Villaseñor, Mendo, \& Losada, 2011). A sua flexibilidade e rigor fazem com que seja considerada a abordagem standard em estudos de observação do comportamento no contexto desportivo (Anguera \& HernándezMendo, 2014), ao permitir a recolha de variáveis categóricas qualitativas e quantitativas (i.e. Mixed Methods) (Anguera, Camerino, Castañer, \& Sánchez-Algarra, 2014; Camerino, Castañer, \& Anguera, 2012). O desenho observacional deste estudo é I/P/M: ideográfico (i.e. centrado na análise de um instrutor), pontual (i.e. cada instrutor é analisado apenas numa aula) e multidimensional (i.e. o comportamento é analisado em simultâneo a partir de diferentes dimensões e categorias) (Anguera et al., 2011). 


\section{Amostra}

Participaram neste estudo 12 instrutores portugueses de Localizada. Esta atividade de fitness, objetiva o desenvolvimento da resistência muscular localizada, através da realização de séries de exercícios, usualmente ao ritmo de música, onde os praticantes utilizam o peso corporal ou outros tipos de materiais (e.g. halteres, caneleiras, barras ou bastões, elásticos) para criar uma carga adicional. Os instrutores foram divididos por dois grupos em função da sua experiência profissional, ou seja, 6 instrutores estagiários (i.e. instrutor-aluno no primeiro ano de experiência profissional) e 6 instrutores experientes (i.e. instrutores com mais de 5 anos de experiência), considerando o modelo de experiência profissional proposto por Berliner (1994): grupo de instrutores experientes - idades compreendidas entre os 29 e os 36 anos $(M=32$; $D P=3$ ), experiência profissional como instrutor de fitness entre os 7 e os 14 anos $(M=9.59 ; D P$ $=2.40)$ e experiência na lecionação da atividade de Localizada entre os 5 e os 14 anos $(M=8.44$; $D P=3.26)$; grupo de instrutores estagiários idades compreendidas entre os 20 e os 26 anos $(M=22 ; D P=2)$, experiência profissional como instrutor de fitness entre os .75 e os .42 anos $(M$ $=.52 ; D P=.11$ ), e experiência na lecionação da atividade de Localizada entre os .75 e os .42 anos $(M=.52 ; D P=.11)$. Foram observadas 12 aulas de Localizada, uma por cada instrutor que participou neste estudo. As sessões foram todas constituídas pelo aquecimento, parte fundamental e retorno à calma e tiveram uma duração média de 43 minutos ( $D P=6$ minutos). Os instrutores tinham a mesma formação académica (i.e. licenciados e estagiários em condição física e saúde no desporto) e ambos os grupos foram constituídos por 3 instrutores do género masculino e 3 instrutores do género feminino.

\section{Instrumentos}

Para a observação do comportamento não verbal cinésico e proxémico dos instrutores de fitness em contexto real de ensino da atividade de Localizada, foram utilizados dois sistemas de observação, desenvolvidos ad hoc, a partir dos sistemas SOCIN e SOPROX (Castañer et al.,
2010, 2013), como é recomendado por Anguera (2003, 2005).

Sistema de Observação da Comunicação Cinésica - Fitness (SOCIN-Fitness) (Alves et al., 2014), o qual é composto por 5 dimensões de análise e 21 categorias (Tabela 1).

Sistema de Observação da Comunicação Proxémica - Fitness (SOPROX-Fitness) (Alves et al., 2013), o qual é composto por 5 dimensões de análise e 23 categorias (Tabela 2).

\section{Procedimentos}

A recolha dos dados foi efetuada sempre mediante um pedido prévio de autorização ao responsável do ginásio, bem como aos instrutores e praticantes envolvidos. Todos os instrutores deram o seu consentimento informado para fazerem parte desta investigação. Os procedimentos adotados cumprem as recomendações éticas definidas por Harriss e Atkinson $(2009,2011)$ para a investigação na área do desporto e exercício, tendo sido adicionalmente aprovados pelo comité de ética e conselho técnico-científico da instituição envolvida com o parecer $n^{\circ} 012 / 2012$.

As gravações dos vídeos (i.e. imagem e som) foram codificadas com recurso ao programa LINCE (Gabin, Camerino, Anguera, \& Castañer, 2012). Previamente foi analisada a qualidade dos dados através do teste das fiabilidades interobservadores e intra-observador em todas as categorias dos sistemas SOCIN-Fitness e SOPROX-Fitness, permitindo garantir que o erro é reduzido e que existe estabilidade e consistência nas observações, assegurando que se pode prosseguir para o registo de dados (BlancoVillaseñor \& Anguera, 2003). Para tal, após o treino dos observadores foi visionado e codificado um vídeo de uma aula de grupo de fitness.

\section{Análise estatística}

Para testar as fiabilidades inter-observadores e intra-observador foi calculado o índice de concordância Kappa de Cohen (Cohen, 1960) para cada categoria de análise em ambos os testes, tendo-se obtido valores de Kappa compreendidos entre .75 e 1, revelando elevados níveis de concordância (Fleiss, 1981).

Para analisar as configurações e sua relação em T-patterns foi utilizado o programa THEME 5.0 (Magnusson, 2000, 2005). 
Tabela 1

Sistema de Observação SOCIN-Fitness

\begin{tabular}{|c|c|c|c|}
\hline Dimensão & Categorias & Código & Descrição \\
\hline \multirow{2}{*}{$\begin{array}{l}\text { Função: Refere-se à função dos gestos, com } \\
\text { intenção comunicativa, que acompanham ou } \\
\text { não o discurso verbal. }\end{array}$} & Regulator & RE & Gestos cujo objetivo é obter uma resposta imediata dos praticantes. \\
\hline & Illustrador & IL & $\begin{array}{l}\text { Gestos que não tem como objetivo obter uma resposta imediata dos praticantes, embora possa } \\
\text { haver resposta num momento futuro. }\end{array}$ \\
\hline \multirow{9}{*}{$\begin{array}{l}\text { Morfologia: Refere-se à forma icónica e } \\
\text { biomecânica do gesto. }\end{array}$} & Emblema Técnico & EMBT & $\begin{array}{l}\text { Gestos com um significado icónico próprio pré-estabelecido, codificados especificamente para o } \\
\text { ensino da atividade e que só têm significado quando aplicados neste contexto. }\end{array}$ \\
\hline & Emblema Social & EMBS & $\begin{array}{l}\text { Gestos com um significado icónico próprio universalmente pré-estabelecido, com caráter } \\
\text { socialmente instituído, não específico da atividade. }\end{array}$ \\
\hline & Emblema Numérico & EMBN & Gestos com um significado icónico próprio pré-estabelecido, que indicam um número. \\
\hline & Deítico & DEI & Gestos que indicam ou apontam para pessoas, segmentos corporais, locais ou objetos. \\
\hline & Pictografico & PIC & Gestos que desenham figuras ou formas no espaço. \\
\hline & Cinetografico & CIN & Gestos que imitam ações ou movimentos no espaço. \\
\hline & Espacial & ESP & Gestos que definem distâncias relativamente a pessoas, objetos e segmentos corporais. \\
\hline & Rítmico & RIT & Gestos que marcam ou definem um ritmo ou velocidade de execução. \\
\hline & Batuta & BAT & $\begin{array}{l}\text { Gestos exclusivos do instrutor, sem significado icónico, que usualmente acompanham e enfatizam } \\
\text { a lógica do discurso verbal. }\end{array}$ \\
\hline \multirow{4}{*}{$\begin{array}{l}\text { Situação: Refere-se à função pedagógica do } \\
\text { gesto no processo de ensino. }\end{array}$} & Informação & INF & Gestos realizados pelo instrutor para informar os praticantes sobre os exercícios. \\
\hline & Feedback & FEED & $\begin{array}{l}\text { Gestos realizados pelo instrutor para ajudar, corrigir os praticantes ou avaliar a sua prestação } \\
\text { motora. }\end{array}$ \\
\hline & Interação & INT & $\begin{array}{l}\text { Gestos realizados pelo instrutor especificamente para encorajar ou interagir em termos relacionais } \\
\text { com os praticantes, seja de forma positiva ou negativa. }\end{array}$ \\
\hline & Organização & ORG & Gestos realizados pelo instrutor para gerir materiais ou organizar os praticantes no espaço. \\
\hline \multirow{2}{*}{$\begin{array}{l}\text { Exercício: Refere-se à participação do } \\
\text { instrutor no exercício quando realiza o gesto. }\end{array}$} & Com Exercício & $\mathrm{CE}$ & O instrutor realiza o exercício. \\
\hline & Sem Exercício & SE & O instrutor não realiza o exercício. \\
\hline \multirow{4}{*}{$\begin{array}{l}\text { Adaptador: Refere-se a gestos em que o } \\
\text { instrutor não tem intenção comunicativa. }\end{array}$} & Objetual & OB & Gestos sem intenção comunicativa, mantendo contato com objetos. \\
\hline & Auto-adaptador & AA & Gestos sem intenção comunicativa, mantendo contato com diferentes partes do seu corpo. \\
\hline & Hetero-adaptador & HA & Gestos sem intenção comunicativa, mantendo contato com outras pessoas. \\
\hline & Multi-adaptador & MUL & Combinações de vários gestos adaptadores definidos anteriormente. \\
\hline
\end{tabular}


Tabela 2

Sistema de Observação SOPROX-Fitness

$$
\text { Dimensão }
$$

Grupo: Refere-se ao número de praticantes para quem o instrutor comunica.

\begin{tabular}{ccl} 
Categorias & Código & Descrição \\
Macro-grupo & MAC & Quando o instrutor comunica com toda a classe. \\
Micro-grupo & MIC & Quando o instrutor comunica com um grupo de praticantes, mas não com toda a classe. \\
Díade & DIA & Quando o instrutor comunica com apenas um praticante. \\
\hline Periférica & P & O instrutor está localizado na zona periférica da sala. \\
Central & C & O instrutor está localizado na zona central da sala. \\
\hline
\end{tabular}

Atitude corporal que revela que o instrutor está ausente do que ocorre na aula, ou que

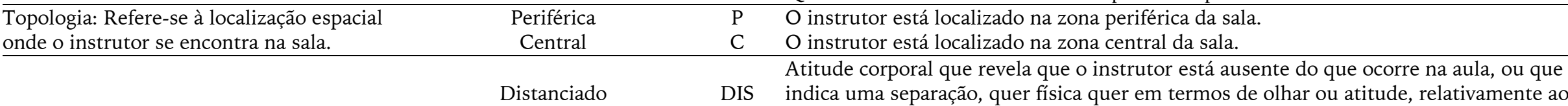

Interação: Refere-se à atitude corporal que indica o grau de envolvimento do instrutor com os praticantes para quem comunica.

Integrado praticantes.

Contacto Táctil

À Frente em Espelho

Orientação: Refere-se à localização espacial do instrutor relativamente aos praticantes, para quem comunica.

\begin{tabular}{cc} 
Atrás & No meio \\
À direita & À esquerda \\
\hline
\end{tabular}

Bípede com Deslocamento

Posição Fixa Bípede

Posição Fixa Sentada

Transição: Refere-se à postura corporal adotada pelo instrutor no espaço.
Posição Fixa Dorsal

Posição Fixa Ventral

Posição Fixa Lateral

Locomoção

Suporte
INT Atitude corporal que revela que o instrutor está envolvido no que se passa na aula, sem existir contacto físico com os praticantes.

Atitude corporal que revela que o instrutor está envolvido no que se passa na aula, existindo contacto físico com os praticantes.

FE O instrutor encontra-se à frente dos praticantes, orientado de frente para o campo de visão dos praticantes.

FC O instrutor encontra-se à frente dos praticantes, orientado de costas relativamente ao campo de visão dos praticantes.

FP O instrutor encontra-se à frente dos praticantes, orientado de lado para estes.

AT O instrutor encontra-se atrás dos praticantes, fora do seu campo de visão.

NM O instrutor encontra-se no meio do espaço ocupado pelos praticantes.

DIR O instrutor encontra-se numa área à direita dos praticantes.

ESQ O instrutor encontra-se numa área à esquerda dos praticantes.

PBD O instrutor está em pé realizando os deslocamentos enquadrados no próprio exercício.

PFB O instrutor está em pé, ou de joelhos, ou agachado, sem se deslocar, embora possa movimentar os seus segmentos corporais.

O instrutor está sentado sem se deslocar, embora possa movimentar os seus segmentos corporais.

PFD O instrutor está deitado dorsal ou em outra posição dorsal sem se deslocar, embora possa movimentar os seus segmentos corporais.

PFV O instrutor está deitado ventral ou em outra posição ventral sem se deslocar, embora possa movimentar os seus segmentos corporais.

PFL O instrutor está deitado lateral ou em outra posição lateral sem se deslocar, embora possa movimentar os seus segmentos corporais.

LOC O instrutor circula pela sala com o objetivo de se deslocar ao longo do espaço.

SU O instrutor está encostado a uma estrutura, material ou pessoa. 
Esta abordagem inovadora no contexto desportivo (Jonsson, Thorsteinsson, \& Tomasson, 2010) permite a recolha de informações sobre a estrutura do comportamento que não podem ser observadas a "olho nu" ou através de qualquer outro método (Magnusson, 2000).

A análise das principais configurações de comportamentos foi realizada através da apresentação do número total de configurações e da frequência absoluta e relativa das 5 configurações mais frequentes, para cada grupo. No caso dos padrões temporais (T-patterns), as estruturas de comportamento detetadas são representadas através de um dendrograma que ilustra um T-pattern de comportamento composto por um conjunto de configurações que ocorrem segundo a mesma ordem e dentro do mesmo intervalo temporal crítico (Jonsson et al., 2010).

Os T-patterns foram detetados através da análise em simultâneo dos seis instrutores de cada grupo, sendo definidos os critérios de inclusão de 3 ocorrências mínimas e um nível de significância de .05. Para cada grupo de instrutores, será apresentado um T-pattern que apresente a estrutura mais completa (i.e. maior número de comportamentos combinados) e que configure um comportamento lógico de atuação profissional.

\section{RESULTADOS}

Das observações realizadas aos 12 instrutores de fitness que participaram neste estudo, foi possível registar um total de 2837 comportamentos cinésicos e respetiva proxémica, considerando os dois grupos de instrutores (Experientes $=1662$; Estagiários $=$ 1175). Cada comportamento observado representa uma configuração de códigos cinésicos e proxémicos registados nesse momento.

\section{Configurações de comportamentos}

Verificou-se a existência de 456 configurações de comportamentos diferentes no grupo de instrutores experientes e 488 para o grupo de instrutores estagiários. $\mathrm{Na}$ Tabela 3 estão apresentadas as cinco configurações mais frequentes obtidas em cada grupo de instrutores e respetivas frequências absolutas, relativas e acumuladas.

Tabela 3

Configuraçôes de comportamentos mais frequentes de cada um dos grupos de instrutores

\begin{tabular}{|c|c|c|c|c|}
\hline Instrutores & Configurações & Freq. $\mathrm{N}^{\mathrm{O}}$ & $\%$ Total & $\%$ Acum. \\
\hline \multirow{5}{*}{ Experientes } & 1ª) RE,DEI,INF,CE,MAC,P,IINT,FC,PFB & 87 & 19.07 & 19.07 \\
\hline & $2^{\mathrm{a})}$ RE,EMBS,INF,CE,MAC,P,IINT,FC,PFB & 82 & 17.98 & 37.05 \\
\hline & 3ª) RE,EMBN,INF,CE,MAC,P,IINT,FC,PFB & 41 & 8.99 & 46.04 \\
\hline & $4^{\mathrm{a})}$ RE,DEI,FEED,CE,MAC,P,IINT,FC,PFB & 36 & 7.89 & 53.93 \\
\hline & 5a) RE,EMBS,FEED,CE,MAC,P,IINT,FC,PFB & 29 & 6.35 & 60.28 \\
\hline \multirow{5}{*}{ Estagiários } & 1ª) IL,DEI,INF,SE,MAC,P,IINT,FE,PFB & 19 & 3.89 & 3.89 \\
\hline & 2a) RE,DEI,INF,CE,MAC,P,IINT,FE,PFB & 19 & 3.89 & 7.78 \\
\hline & $3^{\mathrm{a})} \mathrm{RE}, \mathrm{CIN}, \mathrm{INF}, \mathrm{SE}, \mathrm{MAC}, \mathrm{C}, \mathrm{IINT}, \mathrm{NM}, \mathrm{LOC}$ & 17 & 3.48 & 11.26 \\
\hline & $4^{\text {a) }}$ IL,BAT,INF,SE,MAC,P,IINT,FE,PFB & 16 & 3.27 & 14.53 \\
\hline & 5a) RE,DEI,INF,CE,MAC,P,IINT,FE,PBD & 16 & 3.27 & 17.80 \\
\hline
\end{tabular}

Legenda. Freq. $=$ Frequência; Acum. $=$ Acumulada.

\section{T-patterns dos instrutores experientes}

O T-pattern detetado nos instrutores experientes representa uma combinação de nove configurações diferentes de comportamentos cinésicos e proxémicos (Figura 1).

O padrão de comportamento inicia-se com uma configuração (01) onde os instrutores estão a regular o comportamento dos praticantes (RE) através da realização de um gesto socialmente pré- estabelecido (EMBS) informando-os sobre o que deve ser realizado (INF) e acompanhando-o na realização do exercício (CE). Esse gesto é dirigido para toda a classe (MAC) a partir de uma posição periférica na sala (P), estando os instrutores envolvidos nas atividades (IINT) à frente dos praticantes de costas para os mesmos (FC) numa posição bípede sem se deslocar no espaço (PFB). Nas duas configurações seguintes (02 e 03) os instrutores apenas mudam a morfologias dos gestos, apontando primeiramente para algo (DEI) e realizando em seguida um emblema numérico (EMBS). Na quarta (04) e quintas (5) configurações os instrutores regulam o comportamento dos praticantes através de um gesto deítico (DEI) e cinetográfico (CIN), respetivamente, com o objetivo pedagógico de fornecer feedback (FEED) a todos os praticantes (MAC), mantendo a restante proxémica. Nas configurações seis (06) e sete (07) os instrutores regulam o 
comportamento (RE), mantêm a morfologia cinetográfico (CIN) sendo que, seguidamente realizam um emblema social (EMBS), para fornecer feedback (FEED) individual (DIA). Por último (configurações 08 e 09), os instrutores voltam a regular o comportamento (RE) através de um gestos que identifica o ritmo ou a velocidade dos exercício (RIT) e cinetográfico
(CIN), com o objetivo de informar (INF) aquando da realização do exercício (CE) todos os praticantes (MAC), a partir de uma posição periférica na sala $(\mathrm{P})$, estando os instrutores envolvidos nas atividades (IINT) à frente dos praticantes e de costas para os mesmos (FC) numa posição bípede sem se deslocar no espaço (PFB).

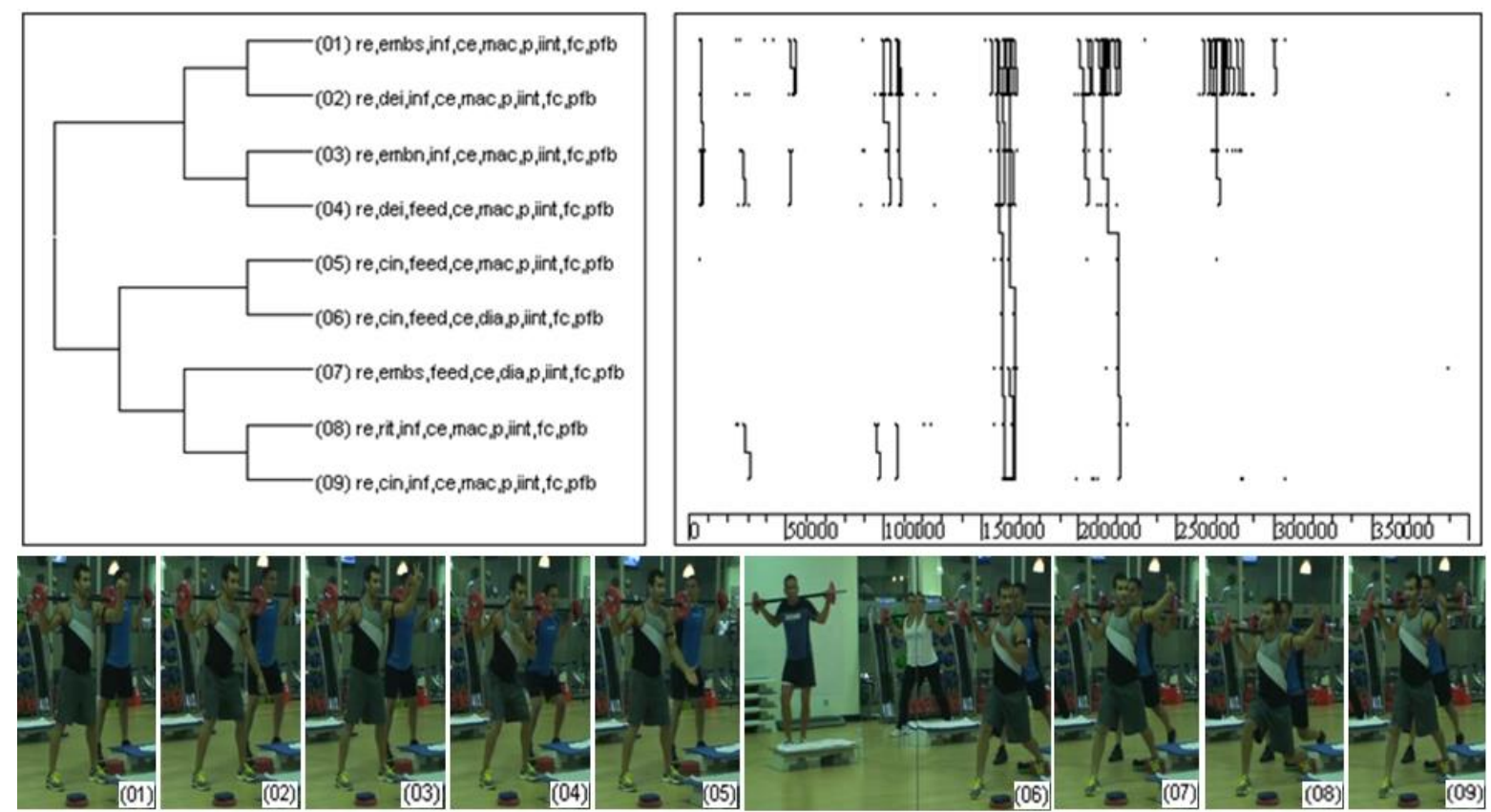

Figura 1. Representação do T-pattern detetado relativamente à comunicação cinésica e proxémica a partir da análise dos seis instrutores experientes da atividade de Localizada

\section{T-patterns dos instrutores estagiários}

Relativamente aos instrutores estagiários, o T-pattern encontrado é composto por quatro configurações de comportamentos cinésicos e proxémicos (Figura 2).

O T-pattern detetado inicia-se com uma combinação (01), onde os instrutores realizam um gesto com uma função ilustrativa, ou seja, não pressupõe uma resposta imediata dos praticantes (IL), através do uso de um gesto de apontar para algo (DEI) com o objetivo de informar (INF) não sendo acompanhado de exercício realizado pelos instrutores (SE). Esse gesto é dirigido para todo o grupo (MAC), estando os instrutores localizados na parte periférica da sala (P), demonstrando estar envolvidos nas atividades (IINT) à frente dos praticantes e de frente para os mesmos (FE) numa posição bípede sem se deslocarem no espaço (PFB). Na configuração seguinte (02) os instrutores realizam novamente um gesto que não pressupõe uma resposta imediata pelos praticantes (IL), sem uma morfologia definida (BAT), com o objetivo de informar (INF), sem estarem a efetuar exercício (SE) e sem alterarem a sua proxémica. Na terceira configuração (03), os instrutores realizam um gesto que não pressupõe uma resposta imediata (IL), através de um emblema socialmente instituído (EMBS), com o objetivo de informar (INF) sem estarem a realizar o exercício (SE), mantendo também a sua proxémica. Na última combinação (04) do padrão, os instrutores realizam um gesto ilustrador, através de um gesto de apontar para algo (DEI) para informar (INF), não estando a realizar exercício (SE). Esse gesto é dirigido para todo o grupo de praticantes (MAC), a partir de uma localização periférica na sala (P), estando os instrutores envolvidos no que se passa na aula (IINT), à frente e com a mesma orientação dos praticantes (FC) estrando em locomoção, ou seja a circular na sala. 

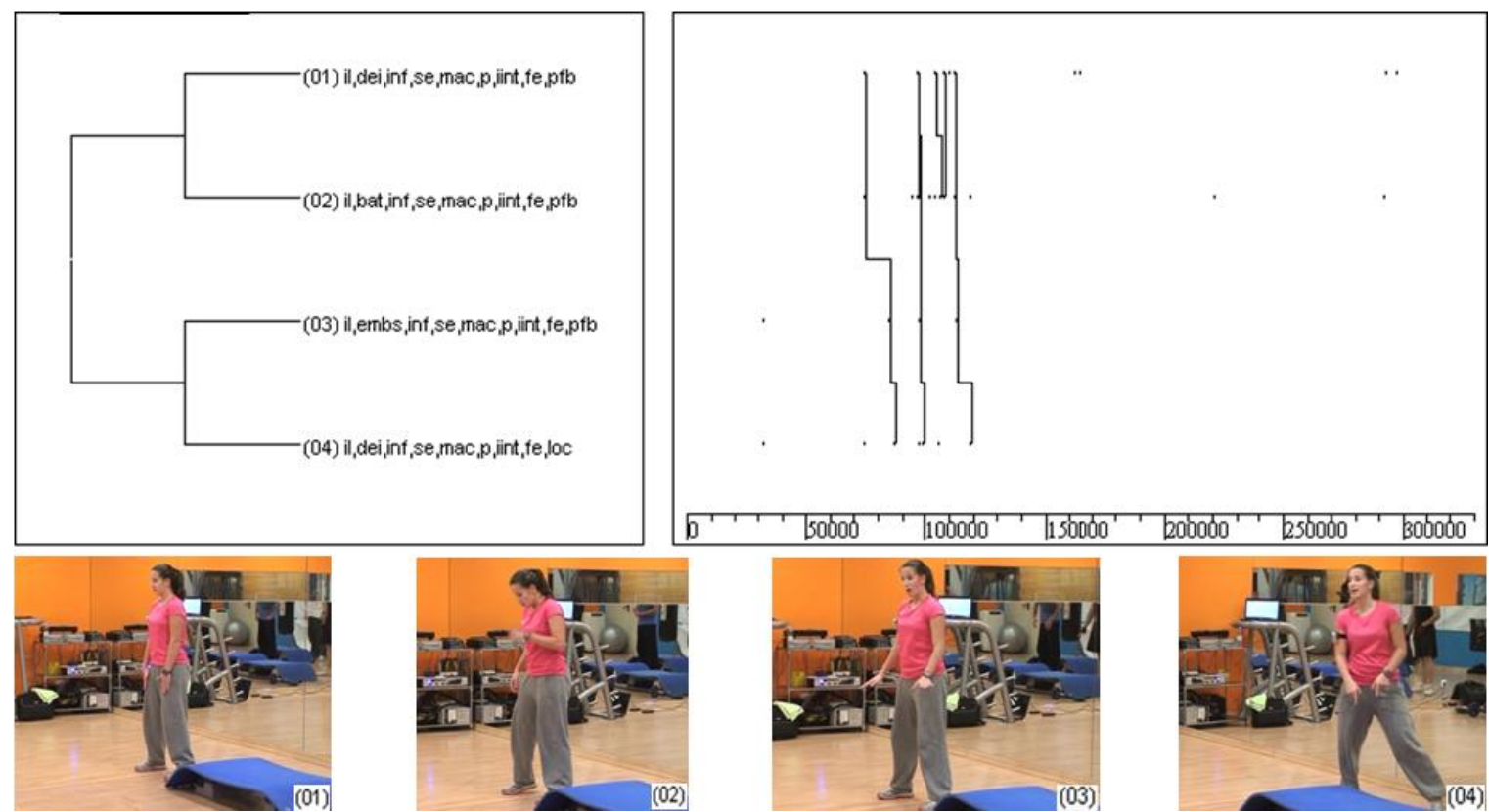

Figura 2. Representação do T-pattern detetado relativamente à comunicação cinésica e proxémica a partir da análise dos seis instrutores estagiários da atividade de Localizada

\section{DISCUSSÃO}

O presente estudo centra-se na análise da comunicação não-verbal dos instrutores de fitness com recurso à metodologia observacional (Anguera, 2003) e objetiva a análise das configurações e T-patterns de comportamento cinésico e proxémico de instrutores com diferentes níveis de experiência profissional, com recurso ao programa informático THEME 5.0 (Magnusson, 2000, 2005). Estudos preliminares realizados à comunicação cinésica (Alves et al., 2014) e proxémica (Alves et al., 2013) de instrutoras de fitness experientes em diferentes atividades de grupo (i.e. Step, Localizada, Indoor Cycling e Hidroginástica) verificaram que o perfil de comunicação não-verbal parece estar relacionado com as caraterísticas próprias de cada atividade. No caso dos instrutores de Localizada verificou-se que comunicaram maioritariamente através de gestos para regular os praticantes, apontando para algo, com o objetivo de informar, aquando da execução dos exercícios em simultâneo com os praticantes. Em termos proxémicos as instrutoras comunicavam maioritariamente para todo o grupo de praticantes, a partir da periferia da sala, com uma atitude corporal que revelava estarem envolvidas com o que se passava na sessão, encontrando-se à frente com a mesma orientação dos praticantes em posição fixa bípede. Estes perfis de comunicação cinésica e proxémica corroboram as configurações de comportamento mais frequentes do presente estudo, e em particular a configuração mais frequente dos instrutores experientes. Adicionalmente, através da análise tabela 3, podemos agora também verificar que as configurações de comportamento de comunicação variam em função da experiência profissional dos instrutores. Os resultados obtidos indicam que os instrutores experientes apresentam uma maior estabilidade de comportamentos cinésicos e proxémicos, já que as cinco configurações mais comuns representaram $60.28 \%$ do total de comportamentos detetados. Por outro lado, os instrutores estagiários apresentam uma maior variabilidade de configurações de comportamentos sendo que as cinco configurações mais frequentes apenas representaram $17.80 \%$ do total dos comportamentos detetados. De acordo com (Berliner, 2001), este automatismo de comportamento verificado nos professores experientes é um mecanismo que lhes permite libertar a atenção para o processamento de informações mais complexas.

A análise da frequência de ocorrências das configurações de comportamentos foi subsequentemente complementada com a informação recolhida ao nível dos T-patterns 
detetados, os quais possuem caraterísticas próprias em cada um dos dois grupos de instrutores analisados:

a) Nos instrutores experientes o T-pattern mais complexo é composto por uma sequência de nove comportamentos, enquanto nos instrutores estagiários o T-pattern mais complexo encontrado representa uma sequência de apenas quatro comportamentos. Tal como verificado anteriormente, o comportamento dos instrutores experientes foi mais estável sendo agora possível também verificar que é mais complexo e estruturado. Esta consistência e estruturação, reflete a complexidade do comportamento que é caraterístico dos professores experientes (Berliner, 2001; Sabers et al., 1991);

b) Os instrutores experientes regulam o comportamento dos praticantes, com o objetivo de obterem uma resposta imediata (i.e. gestos reguladores), enquanto os instrutores estagiários se centram na utilização de gestos ilustradores que não pressupõem essa resposta imediata. Esta diferença de atuação poderá indiciar uma maior preocupação dos instrutores experientes em potenciar o tempo de prática dos praticantes, já que dão as informações enquanto regulam o comportamento, ao passo que os instrutores estagiários fazem-no em situações discursivas e ilustrativas. Note-se que uma das caraterísticas dos professores experientes é a sua maior capacidade para selecionar e estruturar as informações importantes (Berliner, 2001), o que $\mathrm{faz}$ com que despendam significativamente menos tempo em comportamentos de instrução (Behets, 1997);

C) Os instrutores experientes estão sempre a acompanhar os praticantes na realização dos exercícios contrariamente aos instrutores estagiários. Esta diferença indicia uma maior capacidade dos instrutores experientes para utilizarem a sua cinésica corporal como modelo de execução, o que possibilita aos praticantes uma identificação rápida dos exercícios a realizar, bem como da sua correta execução, evitando parar a aula com a verbalização e a ilustração dos exercícios. A utilização o seu corpo como modelo pode servir também como uma estratégia de motivação para fazer com que os alunos se empenhem nos exercícios (Franco, Rodrigues, \& Castañer, 2008);

d) Os instrutores experientes intercalam os comportamentos para informar os praticantes sobre os exercícios (i.e. Informação) com comportamentos para ajudar, corrigir os praticantes ou avaliar a sua prestação motora (i.e. Feedback). Esta combinação não se verifica no comportamento dos instrutores estagiários que apenas dão informação sobre os exercícios. Estes resultados são consistentes com os encontrados por Hogan, Rabinowitz, e Craven (2003), onde se verificou uma tendência para os professores inexperientes se focalizarem em si próprios, estando primeiramente preocupados com a transmissão de informação, ao passo que os professores experientes se focalizavam nas aprendizagens dos alunos, preocupando-se em dar resposta às necessidades sentidas pelos alunos numa dada situação específica. Por outro lado, também é sabido que os professores experientes monitorizam melhor as aprendizagens e as respostas dos alunos aos feedbacks (Berliner, 2001), o que também é evidenciado nos resultados obtidos. Note-se que a atenção recebida e a adequação dos exercícios é um dos aspetos tidos como mais importante pelos praticantes de exercício para a sua avaliação da qualidade do instrutor (Papadimitriou \& Karteroliotis, 2000);

e) Por fim, destaca-se o facto de, nos $T$ patterns identificados, os instrutores experientes utilizaram cinco diferentes morfologias de gestos ao passo que os instrutores estagiários se cingiram à utilização de três morfologias distintas, onde se incluem a utilização de batutas, que representam gestos sem significado icónico, que usualmente acompanham e enfatizam a lógica do discurso verbal. Esta diferença revela uma maior capacidade por parte dos instrutores experientes de tirarem partido da comunicação cinésica gestual, já que utilizam morfologias mais diversificadas. A capacidade de utilização deste tipo de comunicação gestual tem sido identificada como um aspeto importante para a qualidade da comunicação (Cartmill, Beilock, \& Goldin-Meadow, 2012; Maricchiolo, Gnisci, Bonaiuto, \& Ficca, 2009; Maricchiolo, Livi, Bonaiuto, \& Gnisci, 2011). 


\section{CONCLUSÕES}

Os resultados obtidos revelaram a existência de T-patterns de comportamento cinésico e proxémico, próprios de cada grupo de instrutores. Os resultados obtidos sugerem uma maior capacidade por parte dos instrutores experientes de tirarem partido da sua comunicação não-verbal, uma vez que apresentam um comportamento cinésico e proxémico mais consistente e complexo do que os estagiários, caraterizado pela regulação do comportamento dos praticantes, através do acompanhamento na realização dos exercícios a partir da posição correspondente, e da intercalação de comportamentos de informação e de feedback, com recurso à utilização de uma morfologia de gestos mais diversificadas. Esta informação poderá ser útil para guiar e potenciar a intervenção pedagógica dos instrutores de fitness. Por fim, importa ainda referir que os resultados obtidos aplicam-se apenas à atividade de Localizada, o que limita a generalização dos resultados encontrados a outras modalidades. Sendo esta uma primeira abordagem ao estudo da comunicação cinésica e proxémica nas aulas de grupo de fitness será importante prosseguir com investigações futuras que analisem as diferenças neste tipo de comunicação em função do género, das atividades de grupo e da formação dos instrutores.

Agradecimentos:

Nada a declarar.

\section{Conflito de Interesses:}

Nada a declarar.

\section{Financiamento:}

Secretaria de Estado de Investigacion, Desarrollo e Innovacion del Ministerio de Economia y Competitividad. Gobierno Español. Proyecto I+D+I: Observación de la interacción en deporte y actividad física: Avances técnicos y metodológicos en registros automatizados cualitativoscuantitativos. [Referencia: DEP2012-32124].

\section{REFERÊNCIAS}

Alves, S. M., Rodrigues, J. F., Castañer, M., Foguet, O. C., Sequeira, P. J., Carvalhinho, L. A., ... Franco, S. A. (2014). Validação e desenvolvimento de um sistema de observação da comunicação cinésica do instrutor de fitness. Motricidade, 10(1), 7787.

http://doi.org/10.6063/motricidade.10(1).2638
Alves, S. M., Rodrigues, J. J. F., Castañer, M., Foguet, O. C., Sequeira, P. J. R. M. de, Carvalhinho, L. A. D., ... Franco, S. C. A. (2013). Sistema de observação da comunicação proxémica do instrutor de fitness (SOPROX-Fitness): desenvolvimento, validação e estudo piloto. Revista Iberoamericana de Psicología del Ejercicio y el Deporte, 8(2), 281-299.

Anguera, M. T. (2003). Observational Methods (General). Em R. Fernández-Ballesteros (Ed.), Encyclopedia of Psychological Assessment (Vol. 2, pp. 632-637). London: SAGE.

Anguera, M. T. (2005). Microanalysis of T-patterns. Analysis of symmetry/asymmetry in social interaction. Em L. Anolli, S. Duncan, M. Magnusson, \& G. Riva (Eds.), From Genomics to Culture Patterns (pp. 51-70). Amsterdam: IOS Press.

Anguera, M. T., Blanco-Villaseñor, A., Mendo, A. H., \& Losada, J. L. (2011). Diseños Observacionales: Ajuste y aplicación en psicología del deporte. Cuadernos de Psicología del Deporte, 11(2), 6376.

Anguera, M. T., Camerino, O., Castañer, M., \& Sánchez-Algarra, P. (2014). Mixed methods en la investigación de la actividad física y el deporte. Revista de Psicologia del Deporte, 23(1), 123130.

Anguera, M. T., \& Hernández-Mendo, A. (2014). Metodología observacional y psicología del deporte: Estado de la cuestión. Revista de Psicologia del Deporte, 23(1), 103-109.

Behets, D. (1997). Comparison of more and less effective teaching behaviors in secondary physical education. Teaching and Teacher Education, 13(2), 215-224. http://doi.org/10.1016/S0742051X(96)00015-7

Berliner, D. C. (1994). Teacher Expertise. Em A. Pollard \& J. Bourne (Eds.), Teaching and Learning in the Primary School (pp. 73-79). London: Routledge.

Berliner, D. C. (2001). Learning about and learning from expert teachers. International Journal of Educational Research, 35(5), 463-482. http://doi.org/10.1016/S0883-0355(02)000046

Birdwhistell, R. L. (1970). Kinesics and Context: Essays on Body Motion Communication. Philadelphia: University of Pennsylvania Press.

Blanco-Villaseñor, A., \& Anguera, M. T. (2003). Calidad de los datos registrados en el ámbito deportivo. Em A. Hernández-Mendo (Ed.), Psicología del Deporte (Vol. 2, pp. 35-73). Buenos Aires: Efdeportes (www.efdeportes.com).

Bray, S. R., Millen, J. A., Eidsness, J., \& Leuzinger, C. (2005). The effects of leadership style and exercise program choreography on enjoyment and intentions to exercise. Psychology of Sport and Exercise, 6(4), 415-425. http://doi.org/10.1016/j.psychsport.2004.07.00 3 
Camerino, O., Castañer, M., \& Anguera, T. M. (Eds.). (2012). Mixed Methods Research in the Movement Sciences: Case Studies in Sport, Physical Education and Dance. New York: Routledge.

Carron, A., \& Burke, S. (2005). Context and physical activity: The influence of others. Sport and Exercise Psychology Review, 1(2), 23-31.

Cartmill, E. A., Beilock, S., \& Goldin-Meadow, S. (2012). A word in the hand: action, gesture and mental representation in humans and nonhuman primates. Philosophical Transactions of the Royal Society B: Biological Sciences, 367(1585),

http://doi.org/10.1098/rstb.2011.0162

Castañer, M., Camerino, O., Anguera, M. T., \& Jonsson, G. K. (2010). Observing the paraverbal communicative style of expert and novice $\mathrm{PE}$ teachers by means of SOCOP: a sequential analysis. Procedia - Social and Behavioral Sciences, 2(2), 5162-5167. http://doi.org/10.1016/j.sbspro.2010.03.839

Castañer, M., Camerino, O., Anguera, M. T., \& Jonsson, G. K. (2013). Kinesics and proxemics communication of expert and novice PE teachers. Quality \& Quantity, 47(4), 1813-1829. http://doi.org/10.1007/s11135-011-9628-5

Cohen, J. (1960). A Coefficient of Agreement for Nominal Scales. Educational and Psychological Measurement, 20(1), 37-46. http://doi.org/10.1177/001316446002000104

Farrington-Darby, T., \& Wilson, J. R. (2006). The nature of expertise: A review. Applied Ergonomics, $37(1), \quad 17-32$. http://doi.org/10.1016/j.apergo.2005.09.001

Fleiss, J. L. (1981). Statistical methods for rates and proportions (2nd ed.). New York: Wiley.

Franco, S. C. A., Rodrigues, J. de J. F., \& Castañer, M. (2008). Comportamento pedagógico dos instrutores de aulas de grupo de fitness de localizada. Fitness \& performance journal, 7(4), 251-263. http://doi.org/10.3900/fpj.7.4.251.p

Gabin, B., Camerino, O., Anguera, M. T., \& Castañer, M. (2012). Lince: Multiplatform Sport Analysis Software. Procedia - Social and Behavioral Sciences, 46, 4692-4694. http://doi.org/10.1016/j.sbspro.2012.06.320

Hall, E. T. (1966). The Hidden Dimension. New York: Doubleday.

Harriss, D. J., \& Atkinson, G. (2009). International Journal of Sports Medicine - ethical standards in sport and exercise science research. International Journal of Sports Medicine, 30(10), 701-702. http://doi.org/10.1055/s-0029-1237378

Harriss, D. J., \& Atkinson, G. (2011). Update--Ethical standards in sport and exercise science research. International Journal of Sports Medicine, 32(11), 819-821. http://doi.org/10.1055/s-00311287829
Hogan, T., Rabinowitz, M., \& III, J. A. C. (2003). Problem representation in teaching: Inferences from research of expert and novice teachers. Educational Psychologist, 38(4), 235-247. http://doi.org/10.1207/S15326985EP3804_3

Jonsson, G. K., Thorsteinsson, V., \& Tomasson, G. G. (2010). Identification of Vertical and Horizontal Movement Patterns in Cod Behavior. Em Proceedings of the 7th International Conference on Methods and Techniques in Behavioral Research (p. 10:1-10:3). New York, NY, USA: ACM. http://doi.org/10.1145/1931344.1931354

Magnusson, M. S. (2000). Discovering hidden time patterns in behavior: T-patterns and their detection. Behavior Research Methods, Instruments, \& Computers, 32(1), 93-110. http://doi.org/10.3758/BF03200792

Magnusson, M. S. (2005). Understanding social interaction: discovering hidden structure with model and algorithms. Em L. Anolli, S. J. Duncan, M. S. Magnusson, \& G. Riva (Eds.), The Hidden Structure of Interaction: From Neurons to Culture Patterns (pp. 3-22). Amsterdam: IOS Press.

Maricchiolo, F., Gnisci, A., Bonaiuto, M., \& Ficca, G. (2009). Effects of different types of hand gestures in persuasive speech on receivers' evaluations. Language and Cognitive Processes, 24(2), 239266. http://doi.org/10.1080/01690960802159929

Maricchiolo, F., Livi, S., Bonaiuto, M., \& Gnisci, A. (2011). Hand gestures and perceived influence in small group interaction. The Spanish Journal of Psychology, 14(2), 755-764.

Martin, K. A., \& Fox, L. D. (2001). Group and Leadership Effects on Social Anxiety Experienced During an Exercise Class1. Journal of Applied Social Psychology, 31(5), 1000-1016. http://doi.org/10.1111/j.15591816.2001.tb02659.x

Papadimitriou, D. A., \& Karteroliotis, K. (2000). The service quality expectations in private sport and fitness centers: a re-examination of the factor structure. Sport Marketing Quarterly, 9(3), 157164.

Sabers, D. S., Cushing, K. S., \& Berliner, D. C. (1991). Differences among teachers in a task characterized by simultaneity, multidimensionality, and immediacy. American Educational Research Journal, 28(1), 63-88. http://doi.org/10.3102/00028312028001063

Webster, C. A. (2008). A communication perspective of expert and novice teachers' instructional behaviors. Research Quarterly for Exercise and Sport, 79(Sup1), A68. http://doi.org/10.1080/02701367.2008.105994 74

Todo o conteúdo da revista Motricidade está licenciado sob a Creative Commons, exceto quando especificado em contrário e nos conteúdos retirados de outras fontes bibliográficas. 